\title{
Analyzing Infrasound and Seismic Signals Emanating from a Waterborne System Using Canonical Modeling and Analysis Methods
}

\author{
William W. Arrasmith, Everett R. Coots, John V. Olson, and Eric A. Skowbo
}

\begin{abstract}
Infrasound and seismic signals of interest (SOI) have been collected from various sources including men, vehicles, boats and machines during a recent field exercise. The designed experiment specifically located infrasound and seismic sensors to gather the SOIs and process them under the framework of the Infrasound Sensors Network (ISNet). This custom signal gathering, pre-processing, and communication suite allows for the exploitation of SOIs from virtually any type of land, air or waterborne signal source. The on-going development of the ISNet and the subsequent SOI signal processing and analysis is supported by this work.
\end{abstract}

Index Terms - Infrasound, infrasound analysis, infrasound array, infrasound classification.

\section{INTRODUCTION}

The detection, characterization, and classification of infrasound signals has been widely documented in the professional literature with much of the emphasis to date being on environmental signals of interest (SOI) such as earthquakes, volcanic eruptions, and bolides. Much work has also been done to study infrasound signatures of man-made origin such as those of missile launches, fixed and rotary-wing aircraft, ground vehicles, machinery, etc. [1]. Some of the most well documented collaborative work to study man-made SOIs comes from a multi-national, joint scientific and academic organization participating in the monitoring of the Comprehensive Nuclear Test Ban Treaty. This world-wide sensor network consists of over 59 fixed monitoring stations and constantly gathers infrasonic and seismic data to verify compliance by member and non-member nations [2].

While naturally occurring infrasound SOIs are continuously being gathered and analyzed, the advancement of the state of the art in processing man-made SOIs offers a wide range of opportunities. Improved algorithms for the detection, characterization and/or classification phases of the

Manuscript received January 31, 2014; revised March 24, 2014. The ISNet work was initially sponsored by the Defense Advanced Research Projects Agency (DARPA) Strategic Technology Office in 2008.

William. W. Arrasmith is with the Department of Engineering Systems, Florida Institute of Technology, Melbourne, FL. 32901 USA (e-mail: warrasmi@fit.edu)

Everett. R. Coots is with the Harris Corporation, Government Communication Systems Division, 1025 W. NASA Blvd. Melbourne, FL. 32904 USA (e-mail: ecoots@ harris.com)

Eric. A. Skowbo is with the Northrop Grumman Corporation, Research and Technology and Military Aircraft Systems, 2000 W. NASA Blvd. Melbourne, 32904 USA (e-mail: eric.skowbo@ngc.com)

John. V. Olson is with the Geophysical Institute, University of Alaska Fairbanks (e-mail: jvo@gi.alaska.edu) analysis process could all aid in the advancement of the field. This paper focuses on the characterization phase and makes use of empirical test data gathered during a specially designed field experiment using a combination of seismic and infrasound sensors to detect a variety of man-made SOIs from a waterborne source. In conjunction with the field experiment, analytical models having the same array configuration and using simulated input stimulus were developed. The analytical models can be validated by comparing the predicted results with those of the field exercise. This paper presents a brief summary of the field measurements, the corresponding analytical predictions and an analysis of the results of the comparison of the two.

\section{INFRASOUND NETWORK}

\section{A. Overview}

A network of infrasound and seismic sensors was deployed in a field experiment described below. The infrasound sensors network (ISNet) is an end-to-end data acquisition, pre-processing, and communication system. It is composed of sub-systems including infrasound and seismic sensors, array processors, power, a metrology station, and a communication uplink. Representative sensors, the data acquisition system, and the metrology station are shown in Fig. 1.
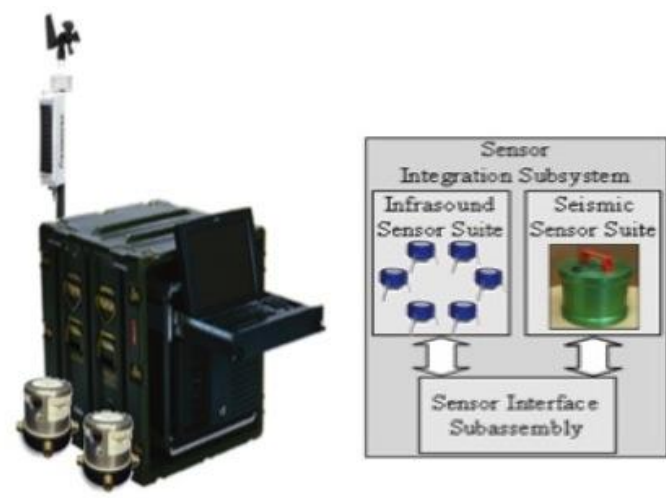

Fig. 1. ISNet representative data acquisition system.

\section{INFRASOUND FIELD EXERCISE}

\section{A. Overview}

A field experiment was conducted in June 2008 for the purpose of gathering infrasound and seismic data on ten different man-made SOIs. The experiment was conducted at Kempfer Ranch (25,000 acres), located in Deer Park, FL between Melbourne and Orlando FL. In the experiment, a 
small motorboat entered a small, simulated lagoon from a nearby canal and docked. Personnel moved equipment out of the boat into a waiting truck. The truck then left the area of the dock and drove to a nearby "village" where men and equipment were off-loaded. The men then created "camp noise" by turning on a gas-powered generator and wood cutting with a chain saw. This wood was then loaded onto the truck and hauled back to the boat dock where the wood and the men boarded the boat. The boat then left the lagoon for the canal. While this experiment was designed to gather data on a variety of man-made SOIs, for this paper we will focus our discussion on the motor boat and its seismic and infrasonic signature. During the experiment the entire flow of men and equipment was exercised two times. Additionally, to ensure sufficient data was collected on the motor boat, the boat made a looping circuit of the lagoon thirty times. This allowed the deployed sensor arrays to gather multiple data sets at a variety of distances and arrival angles as the boat moved around the lagoon. This allowed us to get a rich data set for the boat and also evaluate repeatability of the measurements and sensor network.

\section{B. Sensor Configuration}

The experimental sensor network consisted of a combination of seismic and infrasound sensors (12 infrasound sensors and 2 seismic sensors). The sensors were placed at distances of $3 \mathrm{~m}$ to $192 \mathrm{~m}$ from the closest point of approach (CPA) in a logarithmic spacing to support geo-location and direction finding. Fig. 2 shows the relative arrangement of the sensor network (not to scale).

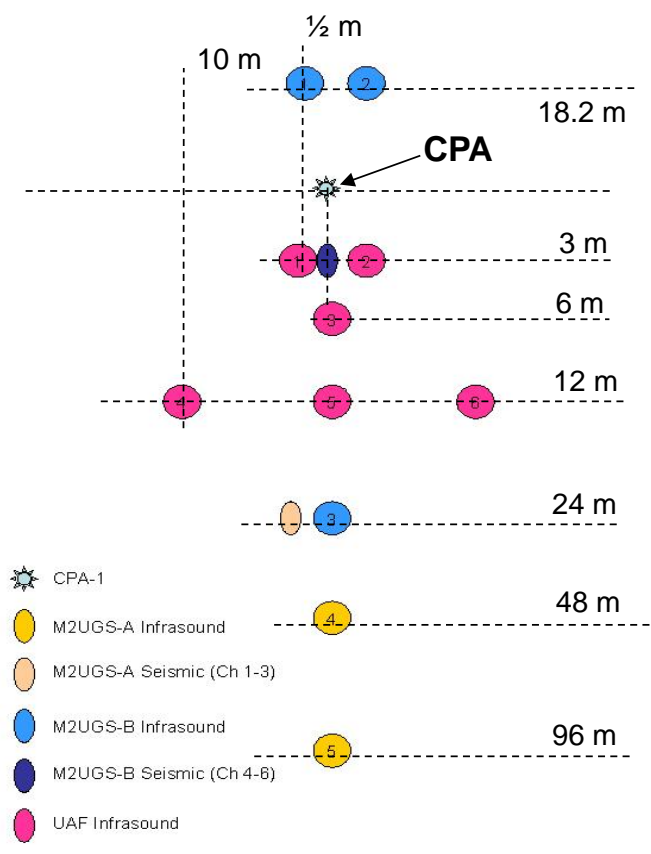

$192 \mathrm{~m}$

Fig. 2. Sensor configuration during experiment.

\section{Observed Results}

As indicated above, the boat made thirty circuits of the experimental course to support extended data gathering. In the case of both the infrasound and the seismic sensors, positive detection of the boat was made at all sensor locations (distances) by analyzing the time-history data [3]. Fig. 3 shows the recorded infrasound array time series and spectrogram plots for one, representative pass of the boat (for clarity) and Fig. 4 shows the recorded seismic data for seven, representative passes of the boat.

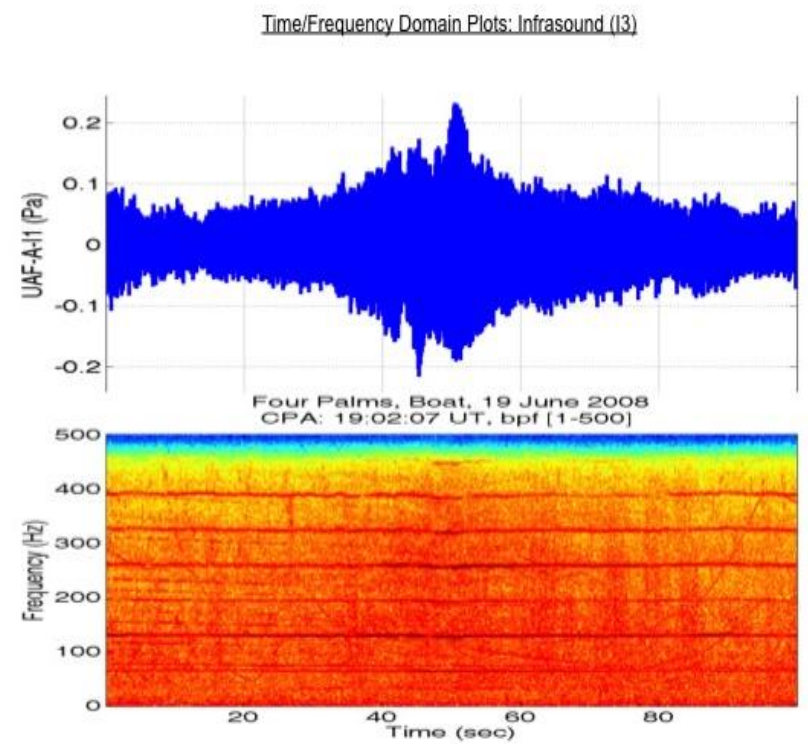

Fig. 3. Infrasound time history for boat.

Infrasound signals were detected that ranged from $\sim 0.25$ $\mathrm{Pa}$ at UAFA-I1 (3m distance) to $\sim 0.01 \mathrm{~Pa}$ at M2UGSA-I6 (192m distance).

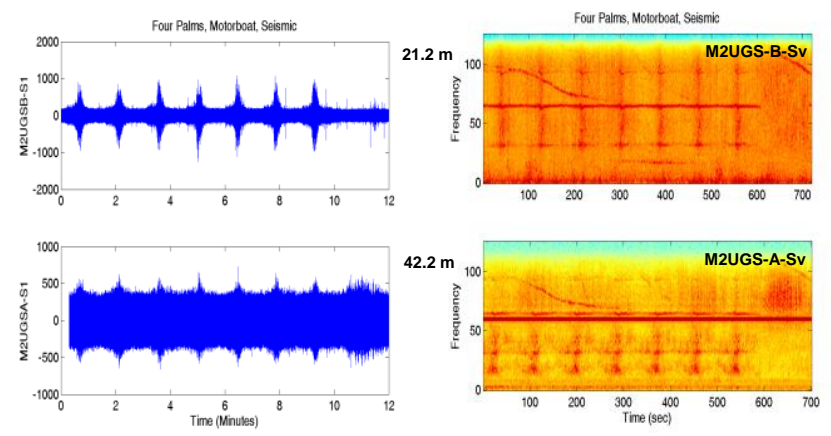

Fig. 4. Seismic time history for boat.

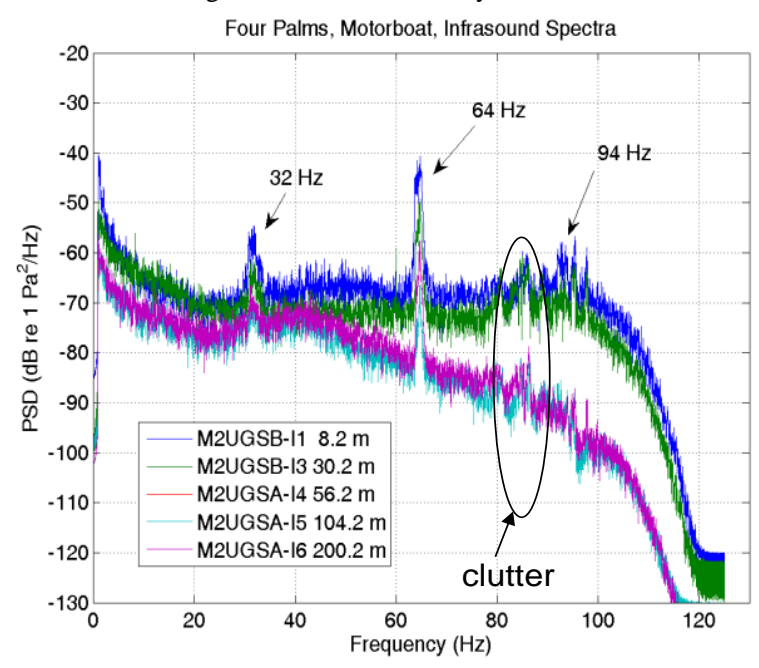

Fig. 5. Infrasound spectra for boat.

Note we show two passes of the boat in the infrasound to illustrate the minimum, repeatable, boat SOI whereas the seismic data illustrates repetition of the SOI over many passes. As can be seen, in both the infrasound and seismic 
data sets, there are observable similarities between the different passes for all infrasound and seismic sensors, especially in the frequency domain.

With detection of the boat successfully demonstrated using both the infrasound and seismic sensors, the data were processed for characterization of the boats unique signature. As Fig. 5 indicates, the boat displayed a $32 \mathrm{~Hz}$ fundamental response with observable harmonics at $64 \mathrm{~Hz}$ and $96 \mathrm{~Hz}$.

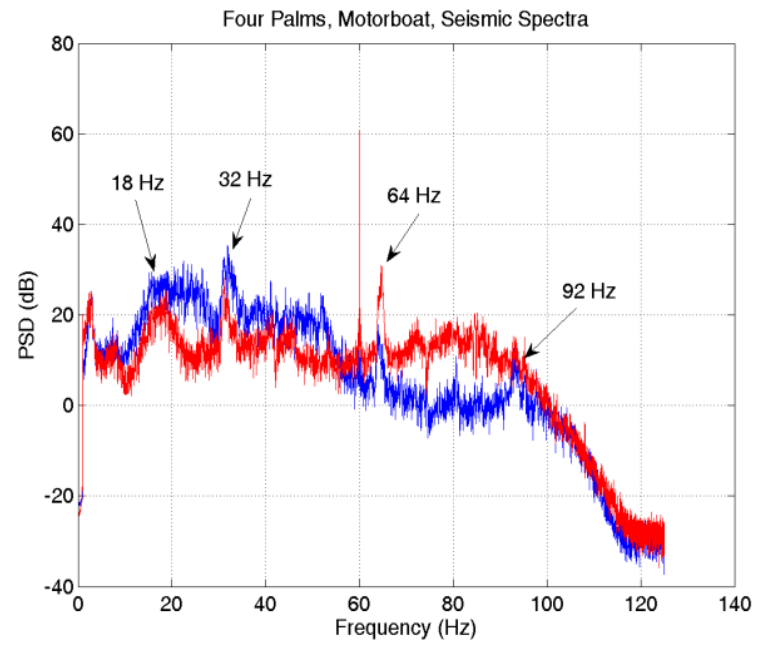

Fig. 6. Seismic spectra for boat.

During all "detection" phases of the experiment the boat was operated with the throttle fully open to ensure a constant sound intensity level. The subject boat used a 25HP, 3 -cylinder outboard motor making 6000 RPM at full throttle. As the fundamental frequency of the boat is determined by the engines composition, speed and propeller rotation we should be able to predict the boats signature (at least for the fundamental) [4]. The projected fundamental frequency for these motor parameters is $150 \mathrm{~Hz}$ [5].

$$
\begin{gathered}
f(H z)=\frac{\left(\frac{\mathrm{RPM}}{60}\right) x(\# \text { Cylinders })}{\# \text { RevolutionsperCycle }} \\
\frac{\left(\frac{6000}{60}\right) x(3)}{2}=150 \mathrm{~Hz} .
\end{gathered}
$$

The above equation provides the fundamental frequency in $\mathrm{Hz}$ for an engine but does not have a load term. Once the engine prop is submerged in water and the boat is under heavy load (as it was in our case), a shift to lower frequencies would be expected. For example, the load on the motor changes the output frequencies. A heavily laden boat would be expected to produce a significantly lower frequency than that of the motor out of the water for instance. Indeed, the observed data of our heavily laden boat shows a fundamental of $32 \mathrm{~Hz}$ - substantially less than the upper limit shown above. We calculated that approximately 1280 RPM are needed to produce a fundamental frequency of $32 \mathrm{~Hz}$ for the above engine not considering load as a factor. Considering uncertainties in the actual RPMs used for the boat passes, and the effect of a heavy load on the engine, the difference between the calculated fundamental frequency and the observed fundamental frequency seem plausible. In any case, the very constant response in the observed data during the detection phase (across multiple circuits of the boat) produced an acceptable data set for this experiment.
The seismic spectra for the boat are presented in Fig.6 and can be compared to the infrasound spectra presented earlier. One can readily identify the $32 \mathrm{~Hz}$ fundamental and $64 \mathrm{~Hz}$ harmonic signatures. The differences between the third harmonic in Fig. 5 and Fig. 6 are due to Doppler shift, the anti-aliasing filters and windowing effects. There also exists an $18 \mathrm{~Hz}$ response that is purely a seismic signal as it does not show up in the infrasound data.

As with most signal processing applications, the use of filtering can improve the dynamic range of the intended signals by reducing or removing the contributions of those that are outside the band of interest. The goal with filtering of the data is to increase the Signal-to-Noise Ratio (S/N or SNR) and improve the interpretability of the data. Without going into a detailed discussion of filter theory (as it is outside the scope of this paper), a simple re-stating of the definition of the signal-to-noise ratio is presented here. For a signal level and a noise level measured over a common test bandwidth and expressed in the $\mathrm{dB}$ power scale, the signal-to-noise ratio (also on a dB scale) is given by:

$$
\left(\frac{S}{N}\right)_{d B}=S N R_{d B}=10 \log _{10}\left(\frac{P_{\text {signal }}}{P_{\text {noise }}}\right)
$$

where the P-terms in Equation 3 are respectively the signal power and the noise power. To demonstrate the potential effectiveness of filtering the test data, infrasound signals from two sensors (M2UGSA-4 and M2UGSA-6) are shown in Fig. 7 with M2UGSA-6 having been run through a Pure-State filter developed by our University of Alaska teammate [6]. The filtered data shows a significant increase in SNR, particularly below $\sim 50 \mathrm{~Hz}$. The SNR near the $32 \mathrm{~Hz}$ response is increased from $\sim 10 \mathrm{~dB}$ to $\sim 30 \mathrm{~dB}$. From a detection standpoint, the benefit of the filtering process is evident when observing the $32 \mathrm{~Hz}$ response. This is difficult to see in the unfiltered data but is quite prominent after the filtering process.

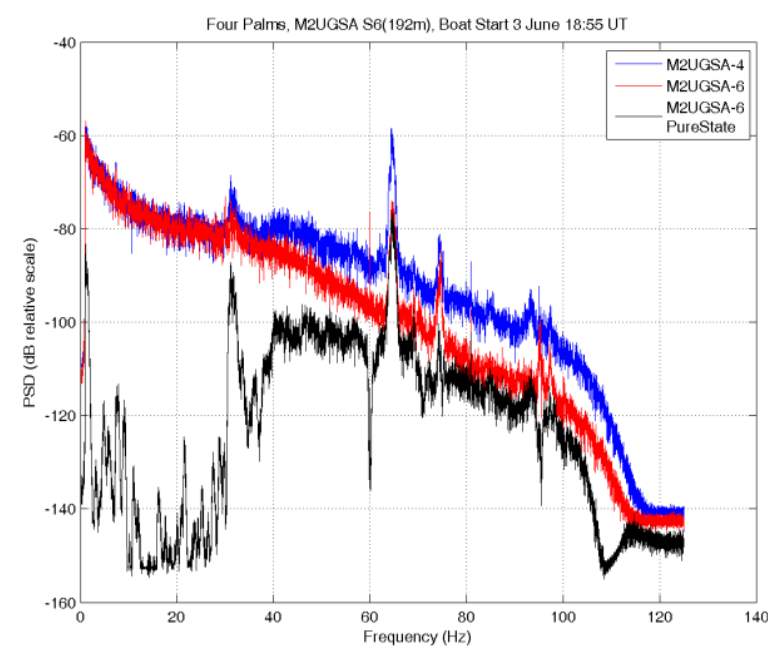

Fig. 7. Infrasound data filtering example.

As with the infrasound data, the seismic data may also be filtered. In this example we filtered the M2UGSA seismic data and present the results in Fig. 8. Similar to the infrasound data, the raw data at the $32 \mathrm{~Hz}$ fundamental has an SNR of $\sim 10 \mathrm{~dB}$, while the filtered response is improved to $\sim 40 \mathrm{~dB}$. Additionally, the response of the $32 \mathrm{~Hz}$ signal is 
easier to detect in the filtered data.

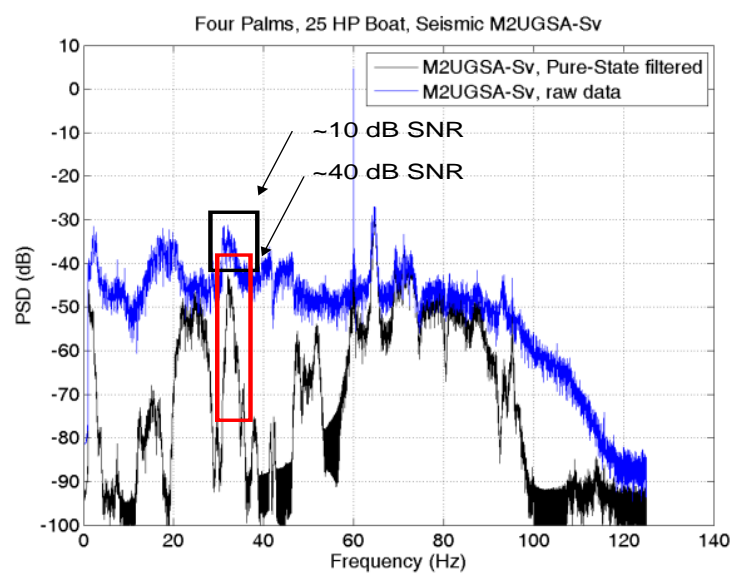

Fig. 8. Seismic data filtering example.

In addition to making the detection easier to observe at the distances used for this experiment, by filtering the data and improving the SNR and thus the dynamic range of the system, the distance at which the boat may be detected can be increased. Fig. 9 presents the projected detection range capability as the signal sound pressure level (SPL) decreases.

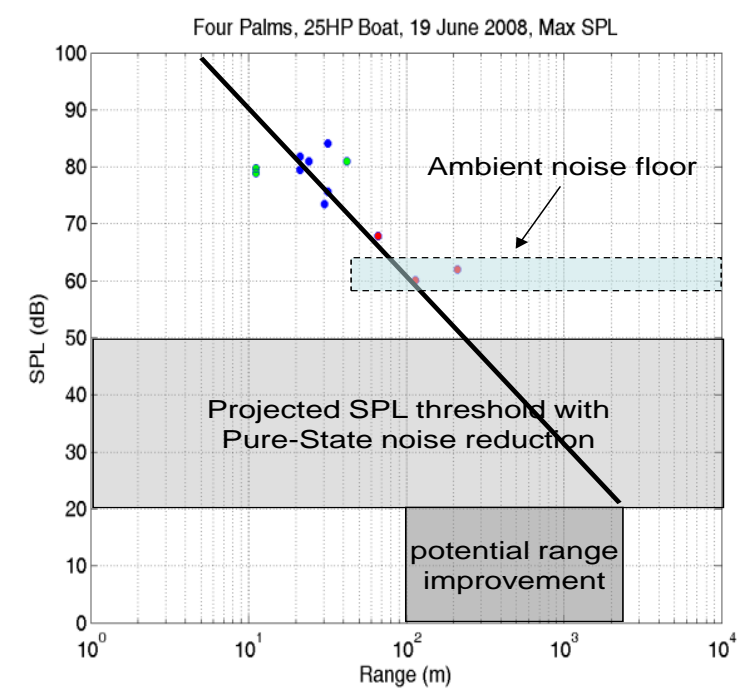

Fig. 9. Infrasound detection capability.

The colored dots on Fig. 9 represent the infrasound sensors placed at the experimental distances from the CPA and the corresponding SPL recorded at each. The ambient noise floor for the experiment ( $\sim 15-20 \mathrm{~dB}$ below the data) is also shown. As described earlier, with the application of Pure-State filtering, the noise floor can be extended an additional 30-40 $\mathrm{dB}$. This increase in dynamic range is represented by the shaded "Projected SPL threshold" region shown in the figure. Recognizing that the SPL falls approximately $30 \mathrm{~dB}$ per decade, a potential increase in the boat detection distance can be predicted. From Fig. 9, when using Pure-State filtering, it should be possible to increase the infrasound detection distance for the boat used in the experiment from $\sim 100 \mathrm{~m}$ to over $1000 \mathrm{~m}$.

\section{Statistical Analysis of Results}

Having the added benefit of multiple sensors in the experiment along with 30 separate trial runs (circuits of the boat) allows for analysis of the data beyond just the infrasound detection and filtering presented above. As the spectrogram below indicates, the boat used in the experiment was detected by multiple infrasound sensors placed at various distances from the CPA. This data can be analyzed to study the effects of distance on detection for example, or the repeatability of the boats signature from one pass to another. Demonstrating strong frequency signature characteristics is a key criterion of any characterization and classifier methodology.

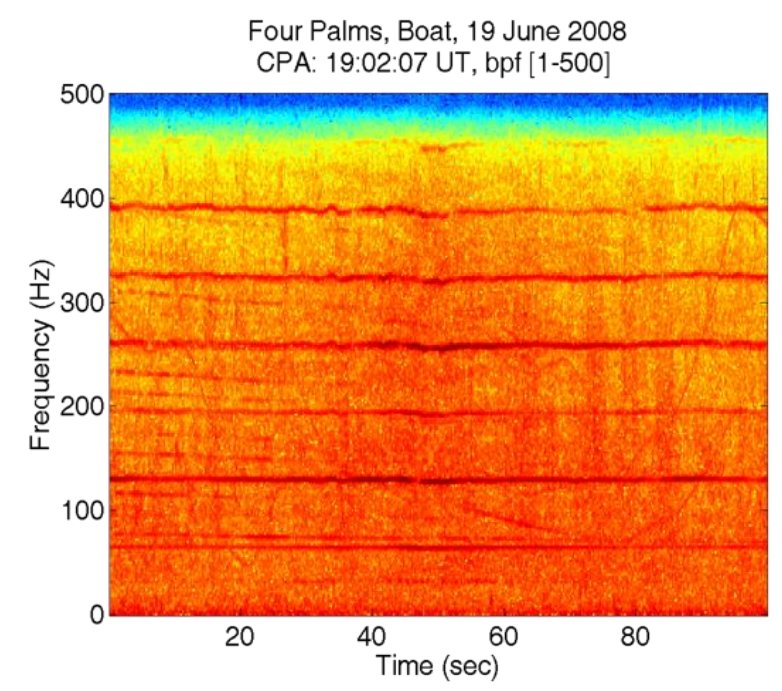

Fig. 10. Boat infrasound signature.

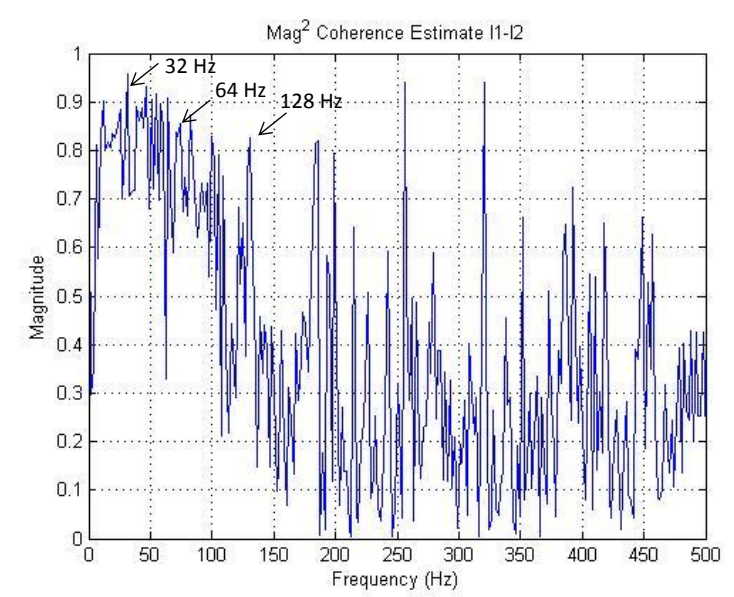

Fig. 11. $\mathrm{Mag}^{\wedge} 2$ coherence estimate I1-I2.

Fig. 10 above represents a snapshot of the boats infrasound signature collected during the experiment (expanded from the bottom of Fig 3 above for clarity). For the following analyses, this dataset was further subdivided by extracting only a representative sample of data. In this case, two consecutive circuits of the boat executed towards the end of the experiment. This resulting data set can be presented as a time-series plot similar to the top of Fig. 3 above or a power spectral density (PSD) plot similar to Fig. 5 above. To evaluate the degradation of the infrasound response as a function of distance from the CPA, we first evaluated the correlation between the spectrums of pairs of sensors. In each case we compared a sensors spectrum to that of the first sensor.

A comparison of Fig. 10 and Fig. 11 above shows the identifiable harmonics detected during the test at $32,64 \mathrm{~Hz}$, 
$128 \mathrm{~Hz}$, and so forth. Fig. 11 indicates a strong coherence between the observed frequency spectra of the sensors \#1 and $\# 2$, particularly for the first $100 \mathrm{~Hz}$. The upper portion of the test bandwidth has a lesser degree of coherence indicating more broadband noise. There are however, clear "spikes" present at the location of the harmonic frequencies. Fig. 12 shows the same type of coherence analysis. In this case sensors \#1 and \#6 are compared. Here there is much less coherence in the lower portion of the test band. While the majority of the band seems to be dominated by broadband noise (uncorrelated), the presence of the key harmonic frequencies can easily be detected.

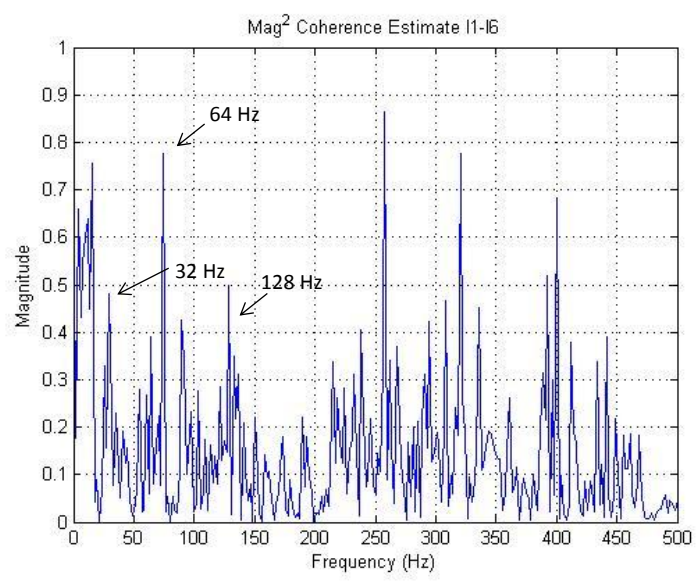

Fig. 12. $\mathrm{Mag}^{\wedge} 2$ coherence estimate I1-I6.

An alternative comparison of the coherence between the infrasound data sets can be made by comparing the data for a given sensor between different circuits of the boat. As indicated earlier, the boat made 30 circuits of the lagoon during the experiment. In this particular case, two of the final circuits are analyzed.

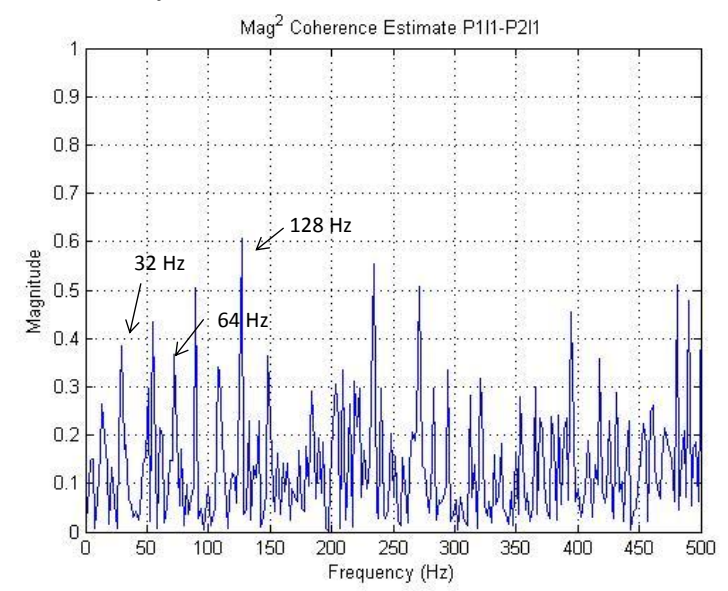

Fig. 13. $\mathrm{Mag}^{\wedge} 2$ coherence estimate I1 pass-pass.

Fig. 13 depicts the magnitude-squared coherence analysis for the infrasound data sets of two independent passes of the boat. The results indicate a very low coherence between the ambient characteristics for the two data sets over the two passes. In the practical sense, apart from the harmonic frequencies, the broad-band signature was not highly correlated between passes. Many of the key harmonic frequency signatures are present in the data however.

Similar to Fig. 13, Fig. 14 above shows a magnitude-squared coherence estimate for two passes of the boat. In the case of Fig. 14 the data presented is from sensor $\# 2$. As with the first sensor, there is very little coherence between the two passes of the boat for ambient conditions. There is however a fairly good correlation between passes for the harmonic frequencies. This indicates that the signals at the fundamental and harmonic frequencies would add coherently between passes and signal averaging methods would be beneficial.

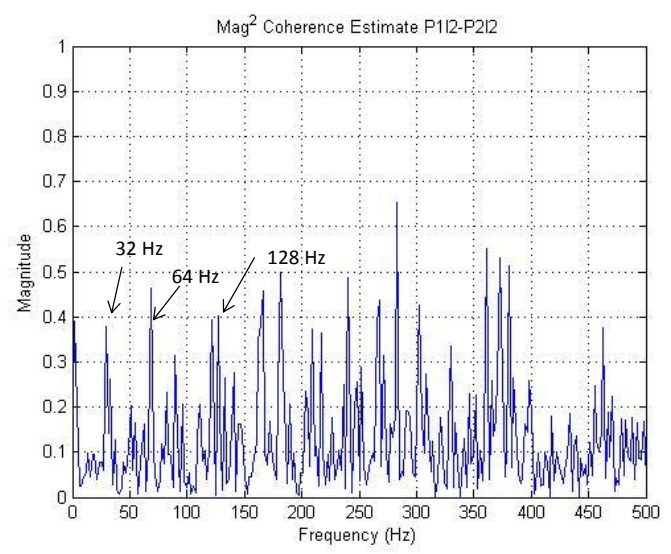

Fig. 14. $\mathrm{Mag}^{\wedge} 2$ coherence estimate I2 pass-pass.

\section{FUTURE WORK}

While this field experiment included SOIs [7] from various sources (men, boats, vehicles, machinery) only the infrasound and seismic data from the boat was analyzed for this paper. Additional work to characterize the SOIs of the other signal sources can be undertaken.

\section{CONCLUSION}

A field experiment has been conducted in which infrasound and seismic signals of interest were collected by an array of sensors. These data were then analyzed to determine the fundamental and harmonic frequency components of the boats characteristic signature. The use of Pure-State filtering improved the SNR of the data (particularly below about $50 \mathrm{~Hz}$ ). Coherence of the signals between sensors for a given pass of the boat as well as signal coherence between passes was analyzed. As would be intuitively expected, the sensor-to-sensor coherence was much higher than that of the signals collected for different passes of the boat.

\section{ACKNOWLEDGMENT}

The authors wish to thank all those who participated in making this endeavor a success. In particular, we would like to thank DARPA/STO for their sponsorship and support. We would also like to thank the team at UAF for their development of the Pure-State Filter, the use of their infrasound array in the experiment, and their critical analytical and planning support. We would like to thank the Northrop Grumman team for their infrasound systems architecture/development/integration/engineering efforts, the planning and execution of the field tests, use of their infrasound/seismic arrays, interacting with stakeholders, and their planning activities. We also would like to thank the FIT team for planning, signal classification, signal processing, 
and analytical support.

\section{REFERENCES}

[1] W. Arrasmith, E. Skowbo, and J. Olson, "An overview of the detection and characterization of man-made signals-of-interest using an infrasound array," INTECH 2013, Budapest, HU, 10-13 September, 2013.

[2] D. N. Green and D. Bowers, "Estimating the detection capability of the international monitoring system infrasound network," J. Geophys. Res., vol. 115,2010

[3] J. V. Olson and C. A. L. Szuberla, Processing Infrasonic Array Data, in Handbook of Signal Processing in Acoustics, Springer Verlag, 2009.

[4] G. Ogden and L. Zurk, "Extraction of small boat harmonic signatures from passive sonar," J. Acoust. Soc. Am, vol. 129, no. 3768, 2009.

[5] C. Greene, and S. Moore, "Man-Made Noise" in Marine, Mammals and Noise, Thomson, Academic Press, NY, 1995, pp. 101-158.

[6] J. C. Samson and J. V. Olson, "Data adaptive polarization filters for multi-channel geophysical data," Geophysics, vol. 46, no. 1423, 1981.

[7] E. A. Skowbo, "Infrasound mobile MASINT Unattended Ground Sensor (M2UGS) system applications and capabilities," Signatures Support Program (SSP) Prospector Working Group, February 2012

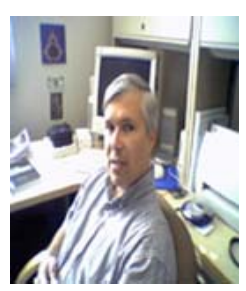

William W. Arrasmith was born in Bad Aibling, Germany on 7 January, 1961. He received his PhD in engineering physics from the Air Force Institute of Technology (AFIT) in Dayton, Ohio in 1995. He earned a MS degree in electrical engineering from the University of New Mexico in Albuquerque New Mexico in 1991. He obtained a BS in electrical engineering from Virginia Tech in Blacksburg, Virginia in 1983.

In his current position, he is a professor of engineering systems at the Florida Institute of Technology (FIT) in Melbourne, Florida, USA. Prior to FIT, he served in the united states air force for over twenty years culminating with a rank of Lt Colonel. During his time in the Air Force, he held several positions including chief, Advanced Science and Technology Division, Applied Technology Directorate at the Air Force Technical Applications Center; assistant professor, Weapons and Systems Engineering Department, United States Naval Academy; program manager, Physics and Electronics Directorate, Air Force Office of Scientific Research; director, Flood Beam Experiment, Air Force Research Laboratory (Kirtland Air Force Base); and project engineer, Teal Ruby Systems Program Office, Dr. Arrasmith is a member of Phi Kappa Phi, Tau Beta Pi, and the American Society of Engineering Education (ASEE) and has two national and one international patent pending. He received the President's Award for Service at Florida Tech in 2013 and the Walter Nunn Excellence in Teaching Award in the College of Engineering at FIT in 2010.

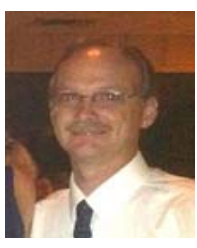

Everett R. Coots was born in 1968, was raised in Montville, CT, and graduated from Florida Institute of Technology, Melbourne, FL in 1993 with a bachelor's degree in electrical engineering, in addition to obtaining a masters-of-science in systems engineering from Florida Institute of Technology in 2007 and a masters-of-science in systems engineering management from Florida Institute of Technology in 2008 . He is currently a doctoral student in systems engineering at Florida Institute of Technology.

He is employed by the Harris Corporation located in Melbourne Florida, as a senior antenna systems engineer in the government communications SYSTEMS Division. Everett has over 20-years of experience in the development of advanced antenna systems including phased arrays, deployable reflectors, and various classical aperture antennas for sea, air, land and space-based applications. Experience includes all levels of the system life-cycle: from preliminary design, to cost estimation and bid/proposal generation, to system integration and test, thru requirements verification and system delivery, to launch and initialization. Mr. Coots current field of study is in infrasound sensor systems, infrasound signal processing and target classification algorithms.

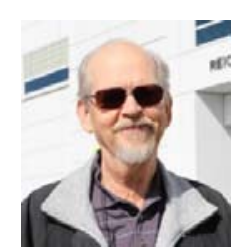

John V. Olson was a professor of physics at the University of Alaska Fairbanks. He obtained a Ph.D. in physics from UCLA in 1970 studying electromagnetic wave propagation in collisionless plasmas.

He worked at the University of Alberta in Edmonton, Canada for nine years as a post-doctoral fellow and research associate. In 1979 he joined the faculty of the University of Alaska Fairbanks (UAF) with a part time appointment at the Geophysical Institute at UAF. He has continued his studies of plasma waves and ionospheric disturbances while at UAF. In 1998 he initiated a program in infrasonic studies as part of the US effort in support of the Comprehensive Nuclear Test-Ban Treaty (CTBT) and has developed an active research group in infrasound studies.

Olson is a member of the American Geophysical Union (AGU), the Acoustical Society of America (ASA), and the IEEE Signal Processing Society.

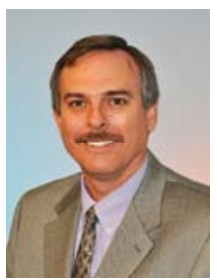

Eric A. Skowbo was born in 1958, was raised in Santa Barbara, CA, and graduated from California State Polytechnic University, Pomona, CA in 1987 with a bachelor's degree in Electrical Engineering, in addition to obtaining a Masters-of-Science in Systems Engineering from Florida Institute of Technology, Melbourne, FL in 2007.

$\mathrm{He}$ is a retired United States Air Force acquisition officer, who led missile development teams for Peacekeeper, Small Missile, and Peacekeeper Rail Garrison programs in addition to completing development and fielding the Air Force Tactical Applications Center Distributed Subsurface Network which was the core of the United States Atomic Energy Detection System. He currently works for Northrop Grumman, located in Melbourne Florida, as Program Manager/Lead Systems Engineer for the Infrasound M2UGS R\&D Group, and leads development of the Infrasound Mobile MASINT Unattended Ground Sensor (M2UGS) System. Eric has over 20 years of experience in the development of MASINT UGS systems, and led the research team that confirmed the hypothesis that UGF can be detected and characterized using unintended infrasonic emissions well outside the "fence-line". An article, Infrasound Sensor System Detection of Atlas V Rocket Launch, was published in the Northrop Grumman Technology Review Journal (Reston, VA: Northrop Grumman Corporation, 2009). Current research emphasis is on infrasound standoff detection and characterization of ground vehicles, rockets, hypersonic test vehicles, underground facilities, vehicle-borne IEDs, unmanned aerial systems, and watercraft using infrasound.

Mr. Skowbo's memberships include the Acoustical Society of America, and the Institute of Electrical and Electronics Engineers. 